\title{
ON LIFTING THE HYPERELLIPTIC INVOLUTION
}

\author{
ROBERT D. M. ACCOLA
}

(Communicated by Albert Baernstein II)

\begin{abstract}
Let $W_{p}$ stand for a compact Riemann surface of genus $p$.
(1) Let $W_{q}$ be hyperelliptic, and let $n$ be a positive integer. Then there exists an unramified covering of $n$ sheets, $W_{p} \rightarrow W_{q}$, where $W_{p}$ is hyperelliptic.

(2) Let $W_{2 n+1} \rightarrow W_{2}$ be an unramified Galois covering with a dihedral group as Galois group, and let $n$ be odd. Then $W_{2 n+1}$ is elliptic hyperelliptic (bi-elliptic).

(3) Let $W_{4} \rightarrow W_{2}$ be an unramified non-Galois covering of three sheets. Then $W_{4}$ is hyperelliptic.
\end{abstract}

\section{INTRODUCTION}

Let $W_{p}$ stand for a compact Riemann surface of genus $p$. All Riemann surfaces in this paper will be compact.

Concerning automorphisms of Riemann surfaces there are two well-known results.

(1) If $W_{p} \rightarrow W_{q}$ is a smooth (unramified) abelian covering of a hyperelliptic $W_{q}$, then the hyperelliptic involution lifts to $W_{p}$ [5].

(2) If $W_{3} \rightarrow W_{2}$ is a (necessarily smooth 2 -sheeted) covering, then $W_{3}$ is hyperelliptic. (This latter result seems to have first appeared in a paper by Enriques [2]. For an interesting proof see Farkas [3].)

In this paper we will discuss two questions.

(1) $W_{q}$ is hyperelliptic, to which (not necessarily Galois) smooth coverings of $W_{q}$ does the hyperelliptic involution lift?

(2) Which of these coverings is itself hyperelliptic?

The kinds of answers given here appear in the abstract.

If $W_{q}$ is hyperelliptic $(q \geq 2)$, then there is a 2-sheeted covering $W_{q} \rightarrow \mathbf{P}^{1}$ branched over $2 q+2$ points in $\mathbf{P}^{1}, a_{1}, a_{2}, \ldots, a_{2 q+2}$. Let $X=\mathbf{P}^{1}-\left\{a_{1}, a_{2}, \ldots, a_{2 q+2}\right\}$. Let $\gamma_{i}$ be the homotopy class in $\pi_{1}(X, \cdot)$ of a curve which "circles" $a_{i}$. Denote $\pi(X, \cdot)$ by $F$, and let $F_{q}$ be the subgroup of index 2 in $\mathscr{F}$ of words in the $\gamma_{i}$ 's where the sum of the exponents is even. In the correspondence between subgroups of $\mathscr{F}$ and coverings of $X, F_{q}$ corresponds to $W_{q}$ punctured at the points above the $a_{i}$ 's. A smooth covering

Received by the editors June 11, 1992 and, in revised form, January 7, 1993.

1991 Mathematics Subject Classification. Primary 14H30; Secondary 30F10. 
$W_{p} \rightarrow W_{q}$ corresponds to a subgroup, $F_{p}$, of $F_{q}$ which contains all the $\gamma_{i}^{2}$ 's and their conjugates in $\mathscr{F}$. We will call such a subgroup smooth. A smooth Galois covering $W_{p} \rightarrow W_{q}$ to which the hyperelliptic involution lifts corresponds to the kernel of a homomorphism $\mu: \mathscr{F} \rightarrow G^{\prime}$ where $G^{\prime}$ is a finite group containing a subgroup $G$ of index 2 where

(i) the kernel of the composition $\mathscr{F} \stackrel{\mu}{\rightarrow} G^{\prime} \rightarrow G^{\prime} / G$ is $F_{q}$; that is, $\mu\left(\gamma_{i}\right) \in$ $G^{\prime}-G$ for all $i$; and

(ii) $\mu\left(\gamma_{i}\right)^{2}=e$ for all $i$.

If $F_{p}$ is the kernel of $\mu$, then the Galois group of the covering $W_{p} \rightarrow W_{q}$, denoted $\mathscr{G}\left(W_{p}, W_{q}\right)$, is isomorphic to $G$. Any involution $T$ in $\mathscr{G}\left(W_{p}, \mathbf{P}^{1}\right)$ with a fixed point will be a lift of the hyperelliptic involution, and $\mathscr{G}\left(W_{p}, \mathbf{P}^{1}\right)$ will be the semidirect product of $\langle T\rangle$ and $\mathscr{G}\left(W_{p}, W_{q}\right)$.

If $W_{p} \rightarrow W_{q}$ is a smooth non-Galois covering, the hyperelliptic involution lifts to $W_{p}$ if there is $\eta \in \mathscr{F}-F_{q}$ so that $F_{p}^{\eta}=F_{p}$ and $\eta^{2} \in F_{p}$. We have the following array of groups:

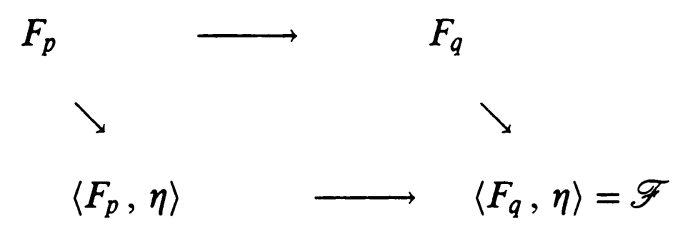

The corresponding array of coverings is $\left(W_{r}\right.$ corresponds to $\left.\left\langle F_{p}, \eta\right\rangle\right)$ :

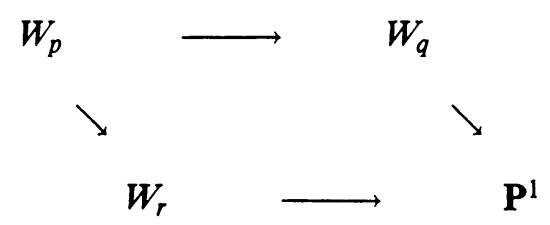

Conjugating $F_{q}$ (resp. $F_{p}$ ) by $\eta$ corresponds to the action of the (resp. lift of the) hyperelliptic involution on $W_{q}$ (resp. $W_{p}$ ) .

Now we shall discuss more systematically the two results mentioned in the second paragraph of this paper.

Lemma 1. If $W_{p} \rightarrow W_{q}$ is a smooth abelian covering and $W_{q}$ is hyperelliptic, then the hyperelliptic involution lifts to $W_{p}$ to be an involution which need not be unique or hyperelliptic. If $g \in \mathscr{G}\left(W_{p}, W_{q}\right)$ and $h$ is a lift of the hyperelliptic involution, then $h^{-1} g h=g^{-1}$. Thus if $\mathscr{G}\left(W_{p}, W_{q}\right)=\langle g\rangle$, then $\langle g, h\rangle$ is a dihedral group, $D_{n}$, of order $2 n$ where $n$ is the order of $g$.

Since we will use Lemma 1 later we now discuss dihedral groups of automorphisms. If $D_{n}=\langle V, R\rangle, V^{2}=R^{n}=V R V R=e$, then $V R^{x}$ is conjugate to $V R^{y}$ if and only if $x-y$ is even. If $n$ is even, there are two conjugacy classes of reflections, and if $n$ is odd, there is only one.

Suppose $W_{p}$ admits a group of automorphisms isomorphic to $D_{n}$. Let $p_{1}$ be the genus of $W_{p} /\langle V\rangle$; let $p_{2}$ be the genus of $W_{p} /\langle V R\rangle$; let $p_{R}$ be the genus of $W_{p} /\langle R\rangle$; and let $p_{0}$ be the genus of $W_{p} / D_{n}$. Then [1]

$$
p+2 p_{0}=p_{1}+p_{2}+p_{R} \text {. }
$$

If $n$ is odd, then $p_{1}=p_{2}$ since conjugate automorphism groups yield conformally equivalent quotients. (Notation. Let $Z_{n}$ stand for the cyclic group of order $n$.) 
Lemma 2. Let $W_{p} \rightarrow W_{q}$ be a smooth cyclic covering of $n$ sheets where $n$ is odd and $W_{q}$ is hyperelliptic. Then the covering $W_{p} \rightarrow W_{q} \rightarrow \mathbf{P}^{1}$ is Galois with $\mathscr{G}\left(W_{p}, \mathbf{P}^{1}\right) \cong D_{n}$. If $V$ is a reflection in $D_{n}$, then the genus of $W_{p} /\langle V\rangle$ is $(n-1)(q-1) / 2$.

Proof. Apply formula (1) since we know $p=n(q-1)+1, p_{R}=q, p_{0}=0$, and $p_{1}=p_{2}$. Q.E.D.

Lemma 3. Let $W_{3}$ be a (necessarily smooth) 2-sheeted covering of $W_{2}$. Then $W_{3}$ is hyperelliptic.

Proof. Apply Lemma 1 and formula (1) with $n=2$. Q.E.D.

\section{SMOOTH HYPERELLIPTIC COVERINGS}

Given that $W_{q}$ is hyperelliptic we consider the problem of a smooth covering $W_{p}$ of $W_{q}$ again being hyperelliptic. If the covering is Galois, the answer was given by Maclachlan [7] and Horiuchi [6].

Lemma 4. If $W_{p} \rightarrow W_{q}$ is a smooth Galois covering where $W_{p}$ is hyperelliptic, then the order of $\mathscr{G}\left(W_{p}, W_{q}\right)$ divides 4 .

If, however, we do not require the covering to be Galois, there is no restriction on the number of sheets in the covering.

Theorem 1. Let $W_{q}$ be a hyperelliptic Riemann surface, and let $n$ be a positive integer. Then there exists a smooth n-sheeted covering $W_{p} \rightarrow W_{q}$ and $W_{p}$ is hyperelliptic.

Proof. If $n=2$, then the result is well known [4, 7], and if $n$ is a power of 2 , the result follows by induction. It remains to prove the theorem for $n$ odd.

We now define a smooth Galois covering $W_{2 p-1} \rightarrow W_{q}$ with Galois group $D_{n}, n$ odd, and $W_{q}$ is hyperelliptic in the context of the fourth paragraph of this paper. To do this we define a homomorphism

$$
\mu: \mathscr{F} \rightarrow Z_{2} \times D_{n} \quad\left(\cong D_{2 n} \text { since } n \text { is odd }\right)
$$

as follows. Let $Z_{2}=\langle C\rangle, D_{n}=\langle V, R\rangle$ as above. Then $\langle C\rangle$ is the center of $D_{2 n}$, and $\langle C V, R\rangle$ is also isomorphic to $D_{n}$.

Let

$$
\begin{aligned}
\mu\left(\gamma_{i}\right) & =C \quad \text { for } i=1,2, \ldots, 2 q-2, \\
\mu\left(\gamma_{2 q-1}\right) & =\mu\left(\gamma_{2 q}\right)=C V, \\
\mu\left(\gamma_{2 q+1}\right) & =\mu\left(\gamma_{2 q+2}\right)=C V R .
\end{aligned}
$$

$\mu$ then extends to a homomorphism onto $D_{2 n}$, and $\mu\left(\gamma_{i}\right)^{2}=e$ for all $i$. The kernel of the composition

$$
\mathscr{F} \rightarrow Z_{2} \times D_{n} \rightarrow\langle C\rangle
$$

corresponds to $W_{q}$ (branch points have been filled in), and the kernel of $\mu$, $F_{2 p-1}$, corresponds to a smooth covering $W_{2 p-1} \rightarrow W_{q}$ with Galois group isomorphic to $D_{n}\left(\cong F_{q} / F_{2 p-1}\right)$. The genus of $W_{2 p-1}$ is $2 n(q-1)+1$. $\mathscr{G}\left(W_{2 p-1}, \mathbf{P}^{1}\right)$ is isomorphic to $Z_{2} \times D_{n}$. In this group of automorphisms let $Z_{2}^{\prime}=\left\langle C^{\prime}\right\rangle$ and let $D_{n}^{\prime}=\left\langle V^{\prime}, R^{\prime}\right\rangle$. (We use primes to distinguish the automorphisms on $W_{2 p-1}$ from the elements of the abstract group $D_{2 n}$.) 
The central involution has branch points above $a_{i}, i=1,2, \ldots, 2 q-2$, so the ramification for the covering $W_{2 p-1} \rightarrow W_{2 p-1} /\left\langle C^{\prime}\right\rangle$ is $2 n(2 q-2)$. By the Riemann-Hurwitz formula the genus of $W_{2 p-1} /\left\langle C^{\prime}\right\rangle$ is one.

Assume $\mathscr{G}\left(W_{2 p-1}, W_{q}\right)$ is $\left\langle V^{\prime}, R^{\prime}\right\rangle$. The other $D_{n}$ is $\left\langle C^{\prime} V^{\prime}, R^{\prime}\right\rangle$ which contains $n$ reflections, all of whose fixed points lie over the $a_{i}$ for $i=$ $2 q-1, \ldots, 2 q+2$.

Thus each such reflection has $4(2 n) / n \quad(=8)$ fixed points. By the RiemannHurwitz formula the genus of $W_{2 p-1} /\left\langle C^{\prime} V^{\prime}\right\rangle$ is $p-2$.

Now consider the four group $\left\{V^{\prime}, C^{\prime}, C^{\prime} V^{\prime}, e\right\} \quad(=H)$. The genera of the quotients with respect to these three involutions are respectively $p, 1$, and $p-2$. By formula (1) the genus of $W_{2 p-1} / H$ is zero. Thus $W_{2 p-1} /\left\langle V^{\prime}\right\rangle$ is hyperelliptic and is a smooth $n$-sheeted covering of $W_{q}$. Q.E.D.

\section{GALOIS COVERINGS}

Lemma 5. Let $W_{p} \rightarrow W_{q}$ be a smooth non-Galois covering where $W_{q}$ is hyperelliptic. Suppose the hyperelliptic involution, $T$, lifts to $W_{p}$. Then the hyperelliptic involution lifts to the Galois closure, $\widetilde{W}$, for the covering $W_{p} \rightarrow W_{q}$.

Proof. Let $T^{\prime}$ be the lift of $T$ to $W_{p}$. Then we have the following array of Riemann surfaces:

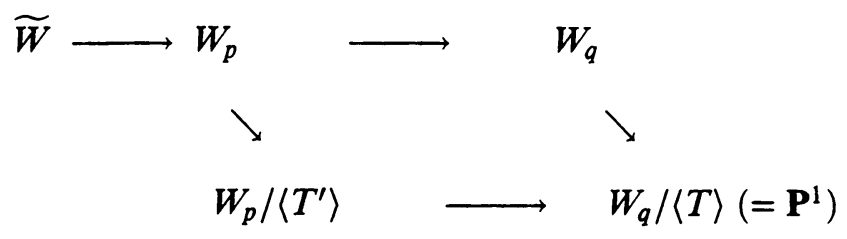

Referring to subgroups of $\mathscr{F}$ (as in the fourth paragraph of this paper), we have the following array of subgroups of $\mathscr{F}\left(\widetilde{F}=\bigcap_{\sigma \in F_{q}} F_{p}^{\sigma}\right)$ :

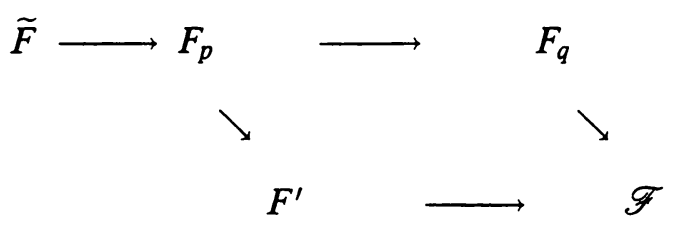

where $F_{q}, F_{p}, F^{\prime}$, and $\tilde{F}$ correspond to $W_{q}, W_{p}, W_{p} /\left\langle T^{\prime}\right\rangle$, and $\widetilde{W}$.

There is an element $\eta \in \mathscr{F}-F_{q}$ so that $F^{\prime}=\left\langle F_{p}, \eta\right\rangle, F_{p}^{\eta}=F_{p}$, and $\eta^{2} \in F_{p}$. Also $\left\langle F_{q}, \eta\right\rangle=\mathscr{F}$, and since $F_{q}$ is normal in $\mathscr{F}, F_{q}^{\eta}=F_{q}$. We now show that $\widetilde{F}^{\eta}=\widetilde{F}$. Since $\widetilde{F}=\bigcap_{\sigma \in F_{q}} F_{p}^{\sigma}$,

$$
\tilde{F}^{\eta}=\bigcap_{\sigma \in F_{q}} F_{p}^{\sigma \eta}=\bigcap_{\sigma} F_{p}^{\eta\left(\eta^{-1} \sigma \eta\right)}=\bigcap_{\sigma} F_{p}^{\eta^{-1} \sigma \eta}=\bigcap_{\sigma} F_{p}^{\sigma}=\tilde{F}
$$

since $\sigma \rightarrow \eta^{-1} \sigma \eta$ is a permutation of $F_{q}$.

Thus $\widetilde{F}$ is normal in $\mathscr{F}$. The Galois covering $\widetilde{W} \rightarrow W_{p} \rightarrow W_{q} \rightarrow \mathbf{P}^{1}$ has branch points of multiplicity 2 corresponding to involutions which are lifts of the hyperelliptic involution of $W_{q}$. Q.E.D.

As an aside from the main arguments of this paper, we consider the situation $W_{p} \rightarrow W_{q} \stackrel{2}{\rightarrow} \mathbf{P}^{1}$, a Galois covering where $W_{p} \rightarrow W_{q}$ is smooth, so the 
hyperelliptic involution lifts. If $H$ is a subgroup of $\mathscr{G}\left(W_{p}, W_{q}\right)$, we ask for conditions on $H$ which assure that the hyperelliptic involution lifts to $W_{p} / H$.

Let $G=\mathscr{G}\left(W_{p}, \mathbf{P}^{1}\right)$, and let $K=\mathscr{G}\left(W_{p}, W_{q}\right)$. Thus $G / K \cong Z_{2}$. For $H \subset K$ let $N_{K}(H)$ be the normalizer of $H$ in $K$ and let $N_{G}(H)$ have similar meaning.

Definition. A subgroup $H$ of $K$ will be called inner if whenever we have an automorphism $\phi$ of $K$, there is $\sigma \in K$ so that $\phi(H)=H^{\sigma}$.

Examples are Sylow subgroups, Hall subgroups of solvable groups, and any subgroup of a group with a trivial outer automorphism group.

Theorem 2. Let $W_{p} \rightarrow W_{q} \stackrel{2}{\rightarrow} \mathbf{P}^{1}$ be a Galois covering where $W_{p} \rightarrow W_{q}$ is smooth. Suppose $H$ is an inner subgroup of $K$.

Then the hyperelliptic involution lifts to $W_{p} / N_{K}(H)$.

Proof. Consider the following array of coverings:

$$
W_{p} / K\left(=W_{q}\right)
$$

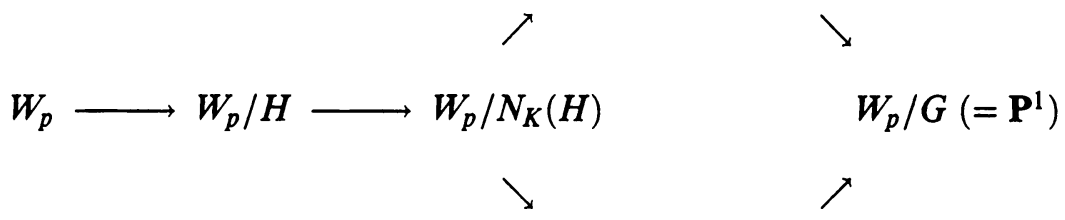

and the corresponding array of groups:

$$
W_{p} / N_{G}(H)
$$

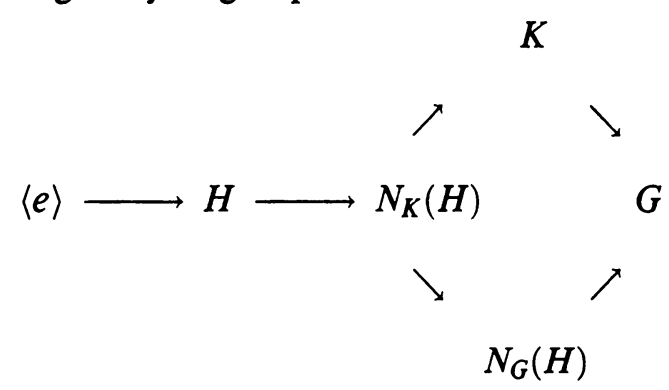

Since $K$ is normal in $G, N_{K}(H)$ is normal in $N_{G}(H)$. Also $N_{G}(H) \cap K=$ $N_{K}(H)$. It now suffices to show that $G=K N_{G}(H)$. To do this let $\sigma \in G$. Since $\theta \rightarrow \theta^{\sigma}$ is an automorphism of $K$ and $H$ is inner, $H^{\sigma}=H^{\eta}$ for some $\eta \in K$. Thus $\sigma \eta^{-1}=n$ where $n \in N_{G}(H)$ and $\sigma=n \eta$. Q.E.D.

The reader may also wish to check that if $H$ is the 2-Sylow subgroups of $K$, then the hyperelliptic involution lifts to $W_{p} / H$.

$$
\text { 4. } q=2
$$

We now assume $q=2$ and use some ideas in the proof of Theorem 1 .

Theorem 3. Let $W_{2 n+1} \rightarrow W_{2}$ be a smooth dihedral covering where $n$ is odd. Then $W_{2 n+1}$ is elliptic hyperelliptic. If $G\left(W_{2 n+1}, W_{2}\right)=\left\langle V^{\prime}, R^{\prime}\right\rangle\left(\cong D_{n}\right)$, then $W_{2 n+1} /\left\langle V^{\prime}\right\rangle$ is hyperelliptic.

Proof. $W_{2 n+1} /\left\langle R^{\prime}\right\rangle \quad\left(=W_{3}\right)$ is a 2-sheeted covering of $W_{2}$ and is hyperelliptic. $W_{2 n+1} \rightarrow W_{3}$ is a cyclic covering, so the hyperelliptic involution on $W_{3}$ lifts to 
$W_{2 n+1}$. We apply Lemma 1 to see that the $2 n$-sheeted covering $W_{2 n+1} \rightarrow W_{3} \rightarrow$ $\mathbf{P}^{1}$ is dihedral. Denote this Galois group $\left\langle U^{\prime}, R^{\prime}\right\rangle$. We have the following array of coverings:

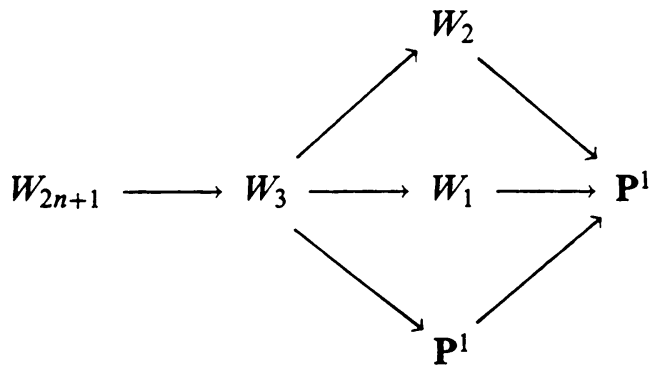

We see that the entire array is Galois with Galois group isomorphic to $Z_{2} \times D_{n}$ $\left(\cong D_{2 n}\right.$ since $n$ is odd). Then central element, $C^{\prime}$, of order 2 in $D_{2 n}$ is unique. We may assume $C^{\prime}=U^{\prime} V^{\prime}$ by replacing $V^{\prime}$ by $V^{\prime} R^{\prime \alpha}$ for suitable $\alpha$.

We are now in the situation considered in the proof of Theorem 1 with $q=2$, $X=\mathbf{P}^{1}-\left\{a_{1}, \ldots, a_{6}\right\}$, and $\mu: \pi_{1}(X, \cdot) \rightarrow D_{2 n}$ where $D_{2 n}=\langle C, V, R\rangle$ and $C V=U$. Since $\mu\left(\gamma_{i}\right)$ has order 2 for all $i$ and all $\mu\left(\gamma_{i}\right)$ 's lie outside one of the $D_{n}$ 's in $D_{2 n}$, say $\langle V, R\rangle$, the possibilities for $\mu\left(a_{i}\right)$ are $C$ and $C V R^{\alpha}$. Since $\langle C V, R\rangle \cong D_{n}$ at least one of the $\mu\left(a_{i}\right)$ 's must be $C$. It follows that two of the $\mu\left(a_{i}\right)$ 's must be $C$. The argument in Theorem 1 now completes the proof. Q.E.D.

Corollary 1. Let $W_{4} \rightarrow W_{2}$ be an unramified 3-sheeted covering.

(a) If the covering is Galois, then $W_{4}$ is elliptic hyperelliptic.

(b) If the covering is not Galois, then $W_{4}$ is hyperelliptic.

Proof. (a) Follows from Lemma 2 with $n=3, q=2$.

(b) The Galois closure of $W_{4} \rightarrow W_{2}$ is $W_{7} \rightarrow W_{4} \rightarrow W_{3}$ where $\mathscr{G}\left(W_{7}, W_{2}\right)=$ $D_{3}$. The result follows from Theorem 3. Q.E.D.

Corollary 2. Let $G$ be a group of order $n$ with a nonnormal subgroup, $H$, of index 3. Suppose $K$ is a subgroup of $H$, normal in $H$, so that $H / K$ is abelian and $\bigcap_{g \in G} g^{-1} K g=\langle e\rangle$. Suppose finally that $W_{n+1} \rightarrow W_{2}$ is an unramified Galois covering with Galois group isomorphic to $G$. Then the hyperelliptic involution lifts to $W_{n+1}$.

Proof. Consider the array of coverings

$$
W_{n+1} \rightarrow W_{n+1} / K \rightarrow W_{n+1} / H \rightarrow W_{2}\left(=W_{n+1} / G\right) .
$$

By Corollary $1 W_{n+1} / H$ is hyperelliptic. The covering $W_{n+1} / K \rightarrow W_{n+1} / H$ is abelian, so the hyperelliptic involution lifts to $W_{n+1} / K$. The covering $W_{n+1} \rightarrow$ $W_{n+1} / H$ is the Galois closure in $G$ of $W_{n+1} / K \rightarrow W_{n+1} / H$. By Lemma 5 the hyperelliptic involution lifts to $W_{n+1}$. Q.E.D.

Examples of such groups are $S_{4}$, the symmetric group of order 24 , and the dicyclic group of order 12.

In the light of Lemma 3 and Corollary 1 one might consider 4-sheeted coverings $W_{5} \rightarrow W_{2}$. The monodromy group, $M$, for this covering is a subgroup of $S_{4}$. If $M=Z_{2} \times Z_{2}, Z_{4}, D_{4}$, or $S_{4}$, then previous results show that the hyperelliptic involution lifts to the Galois closure of $W_{5} \rightarrow W_{2}$, and it follows 
by Theorem 2 that it lifts to $W_{5}$. (If $M=S_{4}$, apply Corollary 2.) If $M=A_{4}$, there is, unfornuately, a counterexample which shows that the hyperelliptic involution need not lift. In cases where it does lift $W_{5}$ is hyperelliptic or elliptic hyperelliptic.

\section{REFERENCES}

1. R. D. M. Accola, Riemann surfaces with automorphism groups admitting partitions, Proc. Amer. Math. Soc. 21 (1969), 477-482.

2. F. Enriques, Sopra le superficie che posseggono un fascio ellittice o di genese due di curve razionli, Roma Reale Acad. Lincei, Rendiconti (5) 7 (1898), 281-286.

3. H. M. Farkas, Automorphisms of compact Riemann surfaces and the vanishing of theta constants, Bull. Amer. Math. Soc. 73 (1967), 231-232.

4. __ Unramified double coverings of hyperelliptic surfaces, J. Analyse Math. 20 (1976), $150-155$.

5. __ Unramified coverings of hyperelliptic Riemann surfaces, Complex Analysis I, Lecture Notes in Math., vol. 1275, Springer-Verlag, New York, 1987, pp. 113-130.

6. R. Horiuchi, Normal coverings of hyperelliptic Riemann surfaces, J. Math. Kyoto Univ. 19 (1979), 497-523.

7. C. Maclachlan, Smooth coverings of hyperelliptic surfaces, Quart. J. Math. Oxford Ser. (2) 22 (1971), 117-123.

Department of Mathematics, Brown University, Providence, Rhode Island 02912

E-mail address: rdma@bromnm.brown.edu 